\title{
The Energy Scale of the Pierre Auger Observatory
}

\section{Bruce R. Dawson ${ }^{* a}$ for the Pierre Auger Collaboration ${ }^{\dagger b}$}

${ }^{a}$ Department of Physics, The University of Adelaide, Adelaide 5005, Australia

${ }^{b}$ Observatorio Pierre Auger, Av. San Martín Norte 304, 5613 Malargüe, Argentina

E-mail: auger_spokespersons@fnal.gov

Full author list: http://www.auger.org/archive/authors_icrc_2019.html

\begin{abstract}
The measurement of energy is one of the most important and challenging tasks of an astroparticle observatory, especially given that the energy must be accompanied by reliable statistical and systematic uncertainties. The energy scale of the Pierre Auger Observatory is based on observations made by its fluorescence detector, taking advantage of the near-calorimetric measurements afforded by this technique. This fluorescence energy calibration is transferred to the surface detector measurements via coincident fluorescence and surface detector observations of air showers at the hybrid observatory. In this contribution, we present the current status of our energy scale. We demonstrate our confidence in the cosmic ray energy assignments by discussing our methods and their systematic and statistical uncertainties. We describe the impact of recent improvements in the estimation of invisible energy, and discuss uncertainties associated with the fluorescence yield, the atmosphere, detector calibration, and the event reconstruction process.
\end{abstract}

36th International Cosmic Ray Conference - ICRC2019

24 July - 1 August, 2019

Madison, Wisconsin, USA

\footnotetext{
* Speaker.

${ }^{\dagger}$ for collaboration list see PoS(ICRC2019)1177
} 


\section{Introduction}

The Pierre Auger Observatory [1] consists of a surface detector (SD) of 1660 water-Cherenkov detector stations spanning an area of $3000 \mathrm{~km}^{2}$ overlooked by 27 large telescopes forming the fluorescence detector (FD). This combination of the SD and the FD provides the advantages of a so-called hybrid observatory. The SD consists of robust detectors that operate 24 hours per day, measuring characteristics of the extensive air shower (EAS) at ground level, including the waterCherenkov detector signal $1000 \mathrm{~m}$ from the shower core, $S(1000)$, the primary energy estimator from the SD. On clear, dark nights the FD observes showers also detected by the SD, providing a near-calorimetric measurement of the primary cosmic ray energy, since the quantity of fluorescence light produced is directly proportional to the total energy deposited by the EAS in the atmosphere. Thus, these hybrid observations calibrate the SD measurement of $S(1000)$ (or more precisely a zenith-angle corrected version of this signal), providing the "energy scale" of the observatory 24 hours per day [2]. While conceptually simple, the definition of the energy scale from the FD observations requires careful measurements and the understanding of many detector and reconstruction effects. Of particular importance are real-time measurements of atmospheric characteristics, including aerosols and clouds [3].

In 2013 we described a comprehensive study of the Auger FD energy scale [4], including a systematic error budget and an estimate of the FD energy resolution. The plan for this contribution is not to update the table of systematic uncertainties, though we plan to publish minor adjustments in the near future. Rather, we will describe the ongoing work in several areas dedicated to crosschecks of assumptions, reduction of model-dependence, incremental improvements to the energy scale, and checks on systematic uncertainties. These activities span both detector and reconstruction effects. We have not discovered any effect that contradicts the 2013 systematic uncertainties in energy, which total $14 \%$.

We have recently completed a review of the FD energy resolution, reported here for the first time. With this quantity and its energy dependence, we can estimate the resolution in the SD energy estimators, important when correcting our SD-based energy spectra for resolution effects [2].

\section{Determining primary cosmic ray energy with the fluorescence technique}

We briefly describe the method for determining the EAS energy using the FD technique, with reference to the energy systematic uncertainties listed in Table 1 . These systematics are estimated for energies above $3 \times 10^{18} \mathrm{eV}$, the full efficiency threshold energy of the $1500 \mathrm{~m}$-spaced SD array. However, the total systematic of $14 \%$ is expected to be approximately valid at lower energies, for example down to an energy of $10^{17} \mathrm{eV}$ relevant for measurements with the $750 \mathrm{~m} \mathrm{SD}$ array [5].

The air shower deposits energy into the atmosphere via ionisation energy losses, and a known fraction of that energy appears as fluorescence light. We use the AIRFLY laboratory measurements [6] of fluorescence yield, including its precise measurement of the emission spectrum, and the fluorescence quenching dependence on atmospheric pressure, temperature, and humidity. The total energy systematic associated with the fluorescence yield is $3.6 \%$.

The isotropically emitted light traverses the atmosphere to reach the FD, typically through larger distances for the rarer, higher energy showers. Energy systematics from the atmosphere include those associated with scattering of light by molecules and aerosols, and those connected to 
the quenching of fluorescence light at the source. The total systematic ranges between $3.4 \%$ and $6.2 \%$ (smaller uncertainty at lower energies), dominated by the uncertainty in aerosol optical depth.

The photometric calibration of the FD telescopes contributes $9.9 \%$ to the energy systematic uncertainty. This includes uncertainties in the absolute calibration at $375 \mathrm{~nm}$ using the end-toend "drum" method performed from time to time, and the nightly relative calibration done with fixed telescope-based light sources [1]. Uncertainty in the wavelength-dependence of the telescope efficiency (including filter, lens, mirror and camera) is also included, one of the systematics checked recently, and reported on below.

Given the measured light as a function of time at the FD, the "shower profile", or energy deposit as a function of atmospheric depth, $\frac{d E}{d X}(X)$, is reconstructed. Several reconstruction uncertainties contribute to an energy systematic of $6.5 \%-5.6 \%$, slightly larger at lower energies. The main contributor is an uncertainty in light collection, given that the image spot is a convolution of the optical point spread function and the finite width of the shower image. Also included are a small systematic associated with the model for multiple-scattered light, and a contribution to account for systematics from the constraints placed on parameters of the Gaisser-Hillas function (see below), mainly important for close-by showers where a smaller range of atmospheric depths is viewed by the FD.

\begin{tabular}{c|c}
\hline \multicolumn{2}{c}{ Systematic uncert. in energy scale } \\
\hline Fluorescence yield & $3.6 \%$ \\
Atmosphere & $3.4 \%-6.2 \%$ \\
FD calibration & $9.9 \%$ \\
FD profile recon. & $6.5 \%-5.6 \%$ \\
Invisible energy & $3 \%-1.5 \%$ \\
Energy scale stability & $5 \%$ \\
\hline TOTAL & $14 \%$ \\
\hline
\end{tabular}

Table 1: Current energy scale systematic uncertainties [4]. A range refers to the change in systematic from $3 \times 10^{18} \mathrm{eV}$ to the highest energies.

The FD technique actually measures the calorimetric energy of the EAS, that portion of the primary cosmic ray energy visible via fluorescence light. A small $(\sim 15 \%)$ correction is applied to the calorimetric energy to get the total energy, accounting for the invisible energy, $E_{\mathrm{inv}}$, associated with high energy muons and neutrinos which deposit most of their energy in the ground. A systematic uncertainty in this correction leads to a systematic in the primary energy of $3 \%-1.5 \%$. Our data-driven approach to estimating $E_{\text {inv }}$ has recently been updated, and is discussed below.

The FD energy scale must be transferred to the SD. Each SD event has an energy estimator $S(1000)$, the detector signal $1000 \mathrm{~m}$ from the shower core [7]. To remove the zenith angle dependence, we convert $S(1000)$ to $S_{38}$, the value it would have if the shower had arrived with the median zenith angle of $\theta=38^{\circ}$. Then, hybrid events are used to fit a relationship between $S_{38}$ and the FD-measured energy, $E_{F D}$, of the form $E_{F D}=A S_{38}^{B}$. In 2013, the statistical error in this fit led to a small contribution to the systematic in the SD energy scale, but given the current event statistics, this contribution is now negligible. However, a related systematic contribution of 5\% is part of Table 1 to account for stability of the energy scale over time. This was estimated by checking the stability of the SD energy, $E_{S D}=A S_{38}^{B}$, for a given $S_{38}$ over a 9-year period.

\section{Examples of checks and improvements to the FD energy scale}

\subsection{Calibration}

Absolute calibrations of the FD telescopes are performed periodically at $375 \mathrm{~nm}$, with nightly 
relative calibrations done at $470 \mathrm{~nm}$ [1]. At the time of the 2013 energy scale definition, the relative spectral response of the telescopes (relative efficiency vs. wavelength) was based on a measurement at a single telescope at 5 wavelengths $(320 \mathrm{~nm}$ to $420 \mathrm{~nm}$ ) using a filtered xenon light source in the drum calibration apparatus. The wavelength range of the spectral efficiency is mostly defined by the band pass of the UV-transmitting optical filters. The efficiency also depends on the transmission of the ring of Schmidt corrector lenses at the aperture, the mirror reflectivity, and the PMT quantum efficiency. We have since improved the measurements of the spectral efficiency, using a drum-mounted monochromator and xenon light source, with measurements over a range from $280 \mathrm{~nm}$ to $440 \mathrm{~nm}$ in $5 \mathrm{~nm}$ steps [8]. Half of the observatory's telescopes are constructed with aluminized glass mirrors, and half with polished aluminium mirrors. The corrector lenses are made of either Borofloat-33 or BK7 glass. Thus, we have measured the relative spectral efficiency for two telescopes each of the four possible combinations of mirror and corrector material. We conclude that the use of these telescope-wise spectral efficiencies, rather than the original five-point calibration, results in cosmic ray energy increases of about $1 \%$ averaged over all telescopes, with one telescope type increasing by $2.5 \%$. These shifts are well within the systematic uncertainty of $3.5 \%$ assigned to the efficiency, as part of the $9.9 \%$ calibration systematic, in the 2013 energy scale study [4]. We note that in [8] we have also established that the relative spectral efficiency is stable with time.

In recent years we have implemented multiple relative calibrations of each telescope during a night, a so-called running calibration. Each camera is illuminated with LED flashes every 30 minutes (with the shutters on the telescope aperture open) as well as at the beginning and end of the nightly run (with shutters closed). This has enabled us to study the nightly variation in calibration associated with the PMT exposure to night sky background light. We conclude that the variations in the relative gain of PMTs during a night are small (up to 2\%) and consistent with the systematic assigned to this in the 2013 study.

Cross-checks of the absolute photometric calibration of the FD telescopes have been achieved using bright U-band stars $[9,10]$. The apparent absolute brightness of a star is measured as a function of the air-mass traversed by the light, allowing for the effect of the atmosphere to be accounted for with this standard astronomical technique. Comparing the estimated star brightness above the atmosphere with the true value yields a check of the calibration. We have confirmed the standard calibration of the FD to a level of approximately $8 \%$, the systematic uncertainty associated with the star technique for a star like Sirius, with a well-known spectrum and apparent magnitude.

\subsection{Atmosphere}

As mentioned, the dominant energy systematic related to the atmosphere is in the determination of the aerosol content. We derive the vertical aerosol optical depth as a function of height, $\operatorname{VAOD}(h)$, from FD observations of the two central laser facilities, the CLF and XLF [11]. Several small improvements have been made to the analysis in recent years, including accounting for the fact that multiple-scattered laser light reaches the FD, and that the quantity of light scattered out of the laser beam is affected by the aerosol content, even though the dominant process is molecular scattering. These improvements, implemented in 2017, led to increases in assigned cosmic ray energies from $1.5 \%$ at $10^{17.5} \mathrm{eV}$ to $3 \%$ at $10^{19.5} \mathrm{eV}$ [12]. More recently, a complete overhaul of the VAOD analysis software (and an integration of it within the observatory's $\overline{\text { Off }} \underline{\text { line }}$ analysis frame- 
work [13]) has allowed us to review all the analysis steps, as well as the statistical and systematic error estimates [3]. The systematics reported in 2013 are still valid.

\subsection{Reconstruction}

In reconstruction of the shower development profile, $\frac{d E}{d X}(X)$, our normal practice is to fit the Gaisser-Hillas $(\mathrm{GH})$ function $[1,14]$ to the measurements. This takes account of energy losses outside the field of view of the FD, since the calorimetric energy estimate, $E_{\mathrm{cal}}$, is the integral of the GH function over all $X$. The GH function has four fitted parameters, namely two shape parameters $X_{0}$ and $\lambda$, the depth of shower maximum $X_{\max }$, and the energy deposit at maximum, $(d E / d X)_{\max }$. In the past, we have fitted these parameters with a procedure that loosely constrains $X_{0}$ and $\lambda$ (tuned using data), and the shower "width", $k=E_{\text {cal }} /(d E / d X)_{\max }$ (tuned using simulations) $[15,16]$. These constraints have little effect on showers with measurements over a large range of depth $X$, but they help minimise systematics for shorter track-length events. However, a long-standing problem, intrinsic to the GH function, is that fitted values of $X_{0}$ and $\lambda$ are strongly correlated.

In 2019 we have implemented a new function for the shower profile, based on the GH function, but with new shape parameters $R$ and $L[17,18]$, namely

$$
f(X)=(d E / d X)_{\max }\left(1+\frac{R}{L}\left(X-X_{\max }\right)\right)^{1 / R^{2}} \exp \left(-\frac{X-X_{\max }}{R L}\right) .
$$

Here $L$ is a measure of the shower profile width, and $R$ is an asymmetry parameter. These two parameters are not correlated, despite them being related to the two parameters $X_{0}$ and $\lambda$ that they replace (see [18]). In our new procedure, we fit the four parameters of the function, with loose constraints on $R(0.257 \pm 0.055)$ and $L\left(\left(227.3+7.44 \log \left(E_{\text {cal }} / \mathrm{EeV}\right)\right) \pm 11.5 \mathrm{~g} / \mathrm{cm}^{2}\right)$. The central values come from fits to well-measured showers, while the $(1 \sigma)$ ranges are determined, from simulations, to be large enough to account for shower-shower fluctuations, and different primary masses. The new procedure reduces reconstructed energies by less than $0.5 \%$ on average, but it has the benefit of fewer constraints and better behaved fits.

\subsection{Invisible Energy}

As mentioned earlier, a portion of the cosmic ray energy is not visible through the fluorescence technique, since that energy is carried by neutrinos and high energy muons that deposit their energy into the ground. Since 2013, we have used data from the observatory to estimate this invisible energy, $E_{\text {inv }}$, rather than using estimates from air shower simulations [19]. In 2019 we have implemented new $E_{\text {inv }}$ estimates, after a detailed study of two alternative methods both using Auger data, one using "vertical" events $\left(\theta<60^{\circ}\right)$ and the other using inclined events $\left(60^{\circ}<\theta<80^{\circ}\right)$ [20]. The methods give consistent results within uncertainties, and are consistent with the 2013 estimates. We now use $E_{\text {inv }}$ from the inclined shower analysis, since it is the most direct of the two methods, given that inclined showers are muon-dominated at ground level. The advantages of using a data-driven estimation of $E_{\text {inv }}$ are that simulations are known to be deficient in their estimation of the muon content of air showers [21] (critical to the invisible energy), and that the estimation naturally takes into account the evolving mass composition of the cosmic rays with energy.

Our $2019 E_{\text {inv }}$ parameterisation covers primary energies $E_{0}$ from $10^{17} \mathrm{eV}$ to $10^{20} \mathrm{eV}$, with values of $E_{\mathrm{inv}} / E_{0}$ ranging from $20 \%$ to $12 \%$, and with systematic uncertainties less than $2 \%$ [20]. 

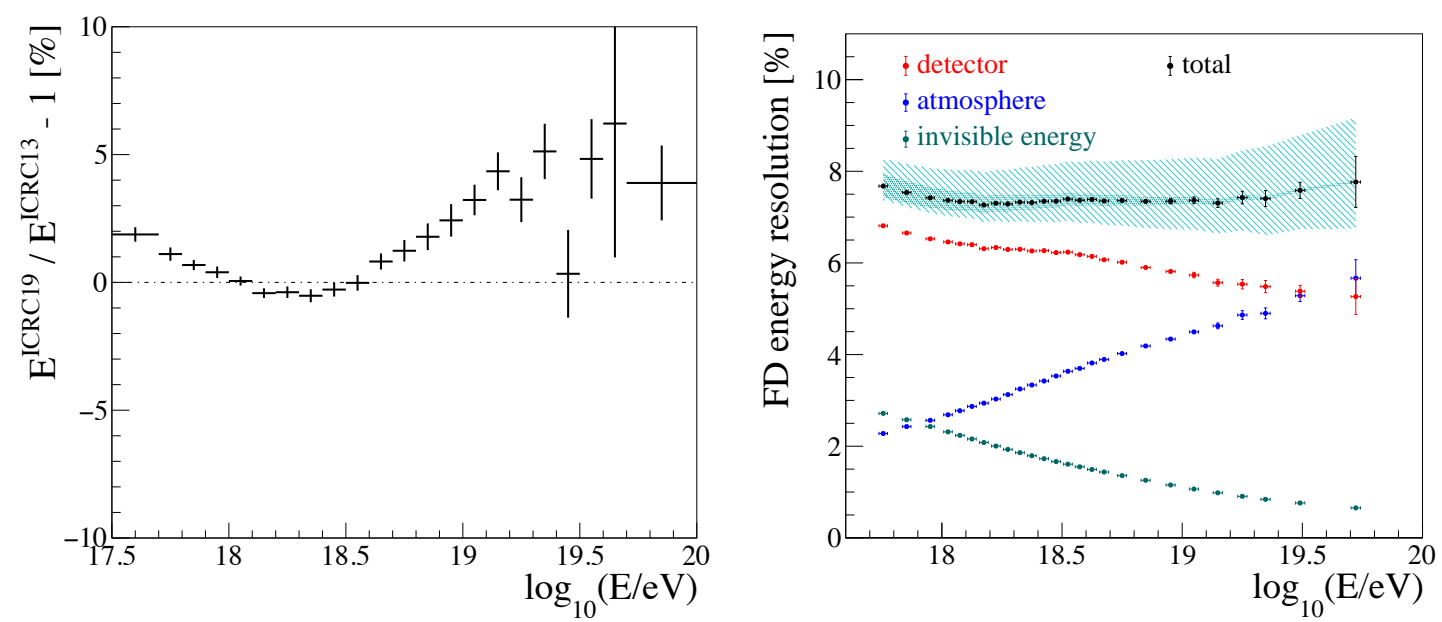

Figure 1: (a): A summary of the FD energy changes for a representative sample of showers, comparing the 2013 and the present FD energy scale. (b): The 2019 FD energy resolution, showing the contributions from the detector (including reconstruction), the atmosphere, and the invisible energy correction. The shaded blue area represents the total systematic uncertainty on the resolution, dominated by the detector/atmosphere, but including the contribution from $E_{\text {inv }}$ (darker shading).

This more extensive study has confirmed the 2013 analysis, and the systematic uncertainties are compatible with the 2013 energy scale error budget in Table 1.

\subsection{Summary of FD energy shifts since 2013}

In Figure 1(a) we show a summary of the small shifts in FD energy assignment that have occurred since 2013, including those from improvements described above. This figure has been derived using a sample of showers already available in 2013, which have been analysed with the reconstruction code used for ICRC 2013 and ICRC 2019.

\section{The FD energy resolution}

We have recently reviewed the assignment of statistical uncertainties to FD-measured energies, and report results here for the first time. We use this FD energy resolution to scale the statistical errors of the SD-measured energies (by requiring a good $\chi^{2}$ for the $E_{F D}=A S_{38}^{B}$ calibration fit), and the deduced SD resolution is used to correct the measured SD energy spectra for resolution effects [2]. The sources of finite FD energy resolution can be divided into three areas - the atmosphere, the detector (including reconstruction), and the invisible energy correction. The study was performed using a large sample of hybrid events with at least three triggered SD stations from the main array, defining an energy range from $10^{17.7} \mathrm{eV}$ to $10^{19.8} \mathrm{eV}$. (A similar study using the $750 \mathrm{~m}$ array, not discussed here, has extended the energy range down to approximately $10^{17} \mathrm{eV}$ ). We show the results in Table 2 and Figure 1(b), and we now explain the contributions.

The atmosphere: We use 3-hourly atmospheric density profiles from the GDAS assimilation model [22]. Fluctuations of pressure, temperature and humidity around the GDAS profiles have been estimated using locally-launched radiosonde balloons, resulting in a 1\% (energy-independent) contribution to the energy resolution. Statistical uncertainties in the aerosol optical depth result from uncertainties in the laser energy and FD photometric calibration, and from the variation in 


\begin{tabular}{c|c}
\hline \multicolumn{2}{c}{ FD energy resolution } \\
\hline Aerosol optical depth & $1.2 \%-3.8 \%$ \\
Horiz. uniform. of aerosols & $1.6 \%-5 \%$ \\
Molecular atmosphere & $1 \%$ \\
\hline Nightly relative calib. & $1.3 \%$ \\
Time drift of FD energies & $2.5 \%$ \\
Mismatch between telescopes & $3.5 \%$ \\
Stat. error from geom. and GH fit & $4.6 \%-2.8 \%$ \\
Extrapolation of profile & $2.2 \%$ \\
\hline$E_{\text {inv }}$ shower-to-shower fluc. & $1.1 \%-0.6 \%$ \\
$E_{\text {inv }}$ mass uncertainty & $2.4 \%-0.3 \%$ \\
\hline TOTAL & $7.6 \%-8.6 \%$ \\
\hline
\end{tabular}

Table 2: New results on the fluorescence detector energy resolution. The table is separated into three sections, referring to contributions from the atmosphere, from the detector and reconstruction, and from invisible energy. A range refers to the change in resolution from $10^{17.7} \mathrm{eV}$ to $10^{19.8} \mathrm{eV}$.

aerosol optical depth across the four quarter-hour measurements in a given hour. These aerosol uncertainties have been reviewed as part of the software overhaul described above, and contribute $1.2 \%$ to $3.8 \%$ to the FD energy resolution, larger at higher energies. Finally, comparing reconstructed energies, and their uncertainties, for "stereo" events (showers seen from two FD sites) has allowed us to estimate an uncertainty that we attribute to non-uniformity of aerosols across the observatory. (Our aerosol measurements, using central laser facilities, probe aerosol content along a limited number of paths.) We estimate this contribution to be from $1.6 \%$ to $5 \%$, again energydependent. The experimental uncertainty of this stereo study (and whether the effect seen is wholly attributable to non-uniformity of aerosols) leads to the dominant contribution to the systematic uncertainty on the total resolution, shown with the blue band in Figure 1(b).

The detector and reconstruction: Detector calibration plays a major role here. Uncertainties include a new estimate of the contribution of the nightly relative calibration (from the new running calibration described above) of $1.3 \%$, and a contribution of $3.5 \%$ that takes into account the small systematic differences in telescope gains across the observatory, determined by studying the ratio $E_{F D} / E_{S D}$ for showers seen by different telescopes. Long-term monitoring of the stability of the telescope gains introduces an uncertainty of $2.5 \%$ to any FD measurement. In the area of reconstruction, formal statistical uncertainties in the shower geometry and the profile function fit (Eq. 3.1) result in a contribution to energy resolution of between $4.6 \%$ and $2.8 \%$. We include an additional $2.2 \%$, derived from simulations, to take into account the uncertainty of the extrapolation of the GH function beyond the viewed profile, something not included in the formal fit error.

The invisible energy correction: This data-driven correction is a fixed function of primary energy, so natural shower-to-shower fluctuations in development, and primary mass variations from the mean mass at a given energy, result in statistical uncertainties in the $E_{\text {inv }}$ correction. We estimate the contribution to energy resolution from shower-to-shower fluctuations to be about $1 \%$, 
with a contribution from mass composition uncertainty ranging from $2.4 \%$ to $0.3 \%$, reflecting the reduction in the spread in mass with energy inferred from $X_{\max }$ measurements [23]. Systematic uncertainties in these estimates lead to the darker-shaded band in Figure 1(b), derived from the uncertainty in the spread of mass, and from assuming either pure proton or pure iron primaries in the estimate of shower-to-shower fluctuations.

The overall FD energy resolution shown in Figure 1(b) takes into account the advantage of "stereo" views of showers at the highest energies. In these cases, the energy used in analyses is the mean of the reconstructed energies (weighted by uncertainties) from the two (or more) stereo views, with the resulting improvement in the statistical error.

\section{Conclusion}

We have reviewed the main systematic uncertainties associated with measuring cosmic ray energy with the Pierre Auger Observatory fluorescence detector. A series of studies have confirmed the total systematic uncertainty of $14 \%$ established in 2013 [4]. A new study of the FD energy resolution has also been described, an important ingredient in evaluating SD energy resolution, and in the correction of energy spectra for resolution effects. The new FD resolution is also consistent with that reported in 2013, but it results from a thorough review where several components have changed in their contributions.

\section{References}

[1] A. Aab et al. [Pierre Auger Collaboration], Nucl. Instrum. Meth. A 798 (2015) 172.

[2] V. Verzi et al. [Pierre Auger Collaboration], POS ( ICRC2019) 450.

[3] V.M. Harvey et al. [Pierre Auger Collaboration], PoS ( ICRC2019) 283.

[4] V. Verzi et al. [Pierre Auger Collaboration], Proc. 33th ICRC 2013, Rio de Janeiro, Brazil [arXiv:1307.5059].

[5] A. Coleman et al. [Pierre Auger Collaboration], PoS ( ICRC2019) 225.

[6] M. Ave et al. [AIRFLY Collaboration], Astropart. Phys. 42 (2013) 90.

[7] D. Mockler et al. [Pierre Auger Collaboration], PoS ( ICRC2019) 353.

[8] A. Aab et al. [Pierre Auger Collaboration], Astropart. Phys. 95 (2017) 44.

[9] A. Segreto et al. [Pierre Auger Collaboration], Proc. CR Intl. Seminar 2018, Nucl. Part. Phys. P. (submitted).

[10] P.H. Nguyen, Ph.D. thesis, The University of Adelaide, http: / / hdl . handle . net/2440/113385 (2018).

[11] P. Abreu et al. [Pierre Auger Collaboration], JINST 8 (2013) P04009.

[12] M. Malacari et al. [Pierre Auger Collaboration], PoS (ICRC2017) 398.

[13] S. Argirò et al., Nucl. Instrum. Meth. A 580 (2007) 1485.

[14] T.K. Gaisser and A.M. Hillas, Proc. 15th ICRC, Plovdiv, Bulgaria 8 (1977) 353.

[15] M. Unger et al., Nucl. Instrum. Meth. A 588 (2008) 433.

[16] F. Fenu et al. [Pierre Auger Collaboration], PoS (ICRC2017) 486.

[17] S. Andringa, R. Conceição and M. Pimenta, Astropart. Phys. 34 (2011) 360.

[18] A. Aab et al. [Pierre Auger Collaboration], JCAP03 (2019) 18.

[19] M. Tueros et al. [Pierre Auger Collaboration], Proc. 33th ICRC 2013, Rio de Janeiro, Brazil [arXiv:1307.5059].

[20] A. Aab et al. [Pierre Auger Collaboration], "Data-driven estimation of the invisible energy of cosmic ray showers with the Pierre Auger Observatory”, Phys. Rev. D (in press) (2019).

[21] A. Aab et al. [Pierre Auger Collab.], Phys. Rev. D 91 (2015) 032003; Errata: Phys. Rev. D 91 (2015) 059901.

[22] P. Abreu et al. [Pierre Auger Collaboration], Astropart. Phys. 35 (2012) 591.

[23] J. Bellido et al. [Pierre Auger Collaboration], PoS (ICRC2017) 506. 